\title{
Note: Tunable notch filter based on liquid crystal technology for microwave applications
}

\author{
V. Urruchi, ${ }^{1, \text { a) }}$ C. Marcos, ${ }^{1}$ J. Torrecilla, ${ }^{1}$ J. M. Sánchez-Pena, ${ }^{1}$ and K. Garbat ${ }^{2}$ \\ ${ }^{1}$ Departamento de Tecnología Electrónica, Universidad Carlos III, Butarque 15, E28911 Leganés, \\ Madrid, Spain \\ ${ }^{2}$ Institute of Chemistry, Military University of Technology, Kaliskiego 2, 00-908 Warsaw, Poland
}

(Received 2 November 2012; accepted 23 January 2013; published online 6 February 2013)

In this work, a compact design of an electrically tunable notch filter, based on liquid crystal (LC) technology, has been designed, manufactured, and characterized. The proposal has been achieved through particular configuration schemes with low cost inverted-microstrip structures and conventional spurlines structures due to its ease of integration. Central frequency tunability has been induced by applying low ac voltages, thus involving low power consumption. For these devices, filter responses have been approached specifically at microwave C-band frequency allocated for many satellite communications applications. Also, it has taken advantage of new highly anisotropic nematic LC mixtures at those frequency ranges. () 2013 American Institute of Physics. [http://dx.doi.org/10.1063/1.4790555]

Recently, liquid crystal (LC) technology has begun to be used in nonoptical applications due to its promising features in further electronic applications ranging from kilohertz to megahertz frequencies. Intrinsic anisotropy of some LC properties, which implies different properties depending of the direction in which they are measured, allows new advanced devices to be designed with tunable features, by using these materials.

The use of LC to design tunable devices at microwave frequency bands is not a new conception; nevertheless there has been an increasing interest in improving their performance, particularly in the last decade. Liquid crystals were recognized as candidates for microwave dielectric substrates in the early 1990s. ${ }^{1}$ Although first approaches to LC devices in waveguides led to bulky and large consumption designs (due to strong magnetic fields for switching LC molecules), most of the recent prototypes, such as tunable phase shifters, ${ }^{2,3}$ capacitors, ${ }^{4}$ filters, ${ }^{5,6}$ or antennas, ${ }^{7}$ have reported practical functions involving electric fields for orienting $\mathrm{LC}$ at those frequencies.

Filters are very valuable devices because they represent a powerful tool for frequency response processing. They are designed in order to select or to remove bands of frequency. A band-rejection filter or band-stop filter is a filter that attenuates a frequency band, while the other frequencies remain unchanged. A notch filter is a band-rejection filter with a narrow stopband, with a high quality factor, that is mainly used to remove spurious frequencies and to filter noise signals.

In this work, the design of a notch filter for about $5 \mathrm{GHz}$ rejection frequency $\left(\mathrm{f}_{0}\right)$, based on the electric field switching of a LC, has been proposed and its feasibility has been demonstrated. Filters working at this frequency, allocated in the C-band, are intended to be used in satellite telecommunication systems or, for example, to avoid the interference

a) Author to whom correspondence should be addressed. Electronic mail: vurruchi@ing.uc3m.es. between the UWB (Ultra Wide-Band) and WLAN (Wireless Local Area Networks) systems. ${ }^{8}$ UWB systems are particularly promising for short-range high-throughput wireless communications.

Multiple solutions have been proposed for providing tunability in microwave devices, such as varactors based on semiconductor technology, micro-electromechanical systems (MEMS), magnetic materials, ferroelectric materials or liquid crystals. The choice of a particular technology depends on the constraints on the specific application. Specifically for space applications, satellites suffer damage of the semiconductor lattice by ionization radiation due to their position. Instead, some LC mixtures have been reported in literature to be stable in the exposure of protons. ${ }^{9}$ Additional favorable features, such as low power consumption and no moving mechanical parts suggest LCs as candidates in these applications. In contrast, insertion losses of LC devices are a key parameter concerning to the device performance not so promising for microwave applications and clear efforts should be focused on further reduction of this feature. Insertion losses include some sources of loss, basically: the dielectric losses (loss tangent), the conductor losses, and additional losses due to the impedance mismatches. A recent detailed study ${ }^{10}$ published in 2010, has reported values of the effective loss tangent for conventional nematic LC devices at the millimeter wavelength range $(30 \mathrm{GHz})$. Loss tangent, in static (from 0.01 to 0.06 ) and dynamic (from 0.04 to 0.001 ) regimes, were, in the worst case, one order of magnitude greater than that of conventional dielectrics such as teflon ${ }^{11}$ and, therefore, were expected to be higher for microwave applications; this constraint has been treated as tolerable low losses.

Manufactured devices were filled with a high dielectric anisotropy LC mixture, so that, the mayor interest of the scheme is its capacity of tuning the rejection frequency. This feature is especially attractive due to the possibility of generating higher tuning frequency ranges than that obtained with conventional nematic LCs. This study is comprised of the 


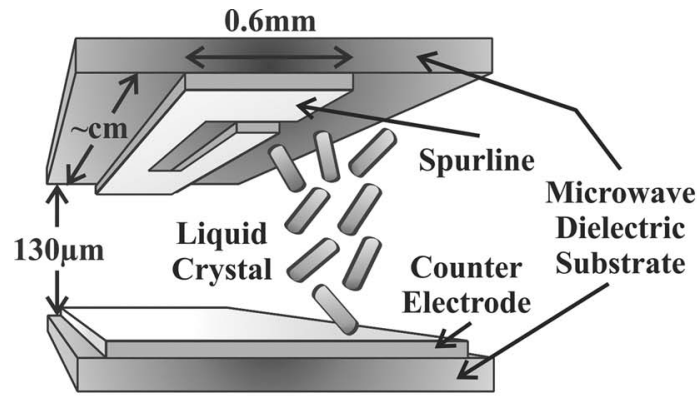

(a)

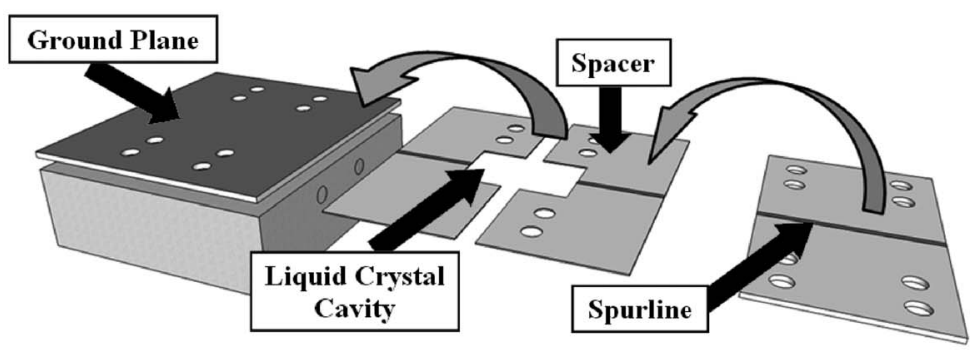

(b)

FIG. 1. Schematic of the notch filter structure based on LC technology. (a) Microstrip inverted arrangement. (b) Detail of the steps for assembling the device.

notch filter design, characterization of its frequency response, measurement of the driving voltage dependence of the filter rejection frequency and finally, analysis and discussion of the voltage tuning dynamic range.

Notch filter of this work is based on inverted microstrip line technology shown in Fig. 1(a). A conventional microstrip transmission line structure has been adapted for manufacturing a device containing a LC mixture inside, thus locating the microstrip line at the inner face of the top substrate. Also, to fitting out a cavity for housing the liquid mixture, an additional spacer and a second bottom substrate (covered with the counter-electrode) are required. Thus, the LC acts as the dielectric substrate of the microstrip line. Figure 1(b) shows the steps for assembling the device. Pattern of the top and bottom electrodes, the microstrip line, and the ground plane, are copper engraved. The microwave dielectric material of the substrates is Taconic TLX-8 with a thickness of $0.8 \mathrm{~mm}$. This material is also used as the spacer, but with a thickness of $130 \mu \mathrm{m}$.

In order to obtain a notch filter characteristic, a spurline structure is used as the microstrip line. A spurline structure is a notch filter widely used in microwave applications as distributed element filter due to its compact design and ease of integration. A typical spurline structure is shown in Fig. 2. It consists of a microstrip line based on two asymmetrical coupled lines, where one of the resonating lines is open-ended. The spurline length $\left(1=\lambda_{0} / 4\right)$ is one quarter the vacuum wavelength, $\lambda_{0}$. It works as a rejection-band filter with a fixed rejection frequency $\left(\mathrm{f}_{0}\right)^{12}$ defined as

$$
f_{0}=\frac{c}{4 \times l \times \sqrt{\varepsilon_{r}}}
$$

where $\mathrm{c}$ is the velocity of light, 1 the spurline length, and $\varepsilon_{\mathrm{r}}$ the permittivity of the microstrip line substrate. For a fixed length of the spurline, the rejection frequency only depends on the permittivity of the substrate.

Taking advantage of the electrically controlled permittivity of LC materials, the filter design can improve strongly

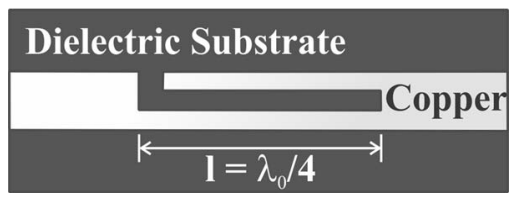

FIG. 2. Shape of the spurline structure acting as the microstrip line. compared to conventional microwave filters. The frequency response of the new filters, with emphasis on the generation of analogue adaptive rejection frequencies, provides enormous versatility on the device.

Positions of LC molecules inside the cavity are conditioned by the alignment protocol of the device. Both electrodes are covered by a rubbed polyimide alignment layer for providing a homogeneous alignment to the device and glass substrates are antiparallel assembled. Without applying any voltage to the LC, molecules place in a near parallel to the glasses position. Effective permittivity of the whole structure in that situation is $\varepsilon_{\mathrm{r} \perp}$ for the input microwave signal. By tuning the LC device with an external ac voltage, molecules tend to follow the field and rotate changing the permittivity between two extreme values corresponding to $\varepsilon_{\mathrm{r} \perp}$ and $\varepsilon_{\mathrm{r} \|}$, respectively. Molecules are placed in a near perpendicular position to the microstrip line when the permittivity is $\varepsilon_{\mathrm{r} \|}$.

A high-birefringence material $\left(\Delta \mathrm{n}=0.3259\right.$ at $20^{\circ} \mathrm{C}$, $589 \mathrm{~nm}$ ) has been used in this work, in order to achieve a higher tunability of the notch filter. It is a nematic LC mixture, 1631F, synthesized in Military University of Technology ${ }^{13,14}$ and composed of fluorosubstituted alkyltolane and alkylphenyltolane isothiocyanates. This material presents the nematic phase from 0 to $102.5^{\circ} \mathrm{C}$. The lateral isothiocyano group induces a large positive dielectric anisotropy $\left(\Delta \varepsilon=9.51\right.$ at $\left.20^{\circ} \mathrm{C}, 10 \mathrm{kHz}\right)$. Unsubstituted cyclohexylbenzene and bicyclohexyl benzene isotiocyanates, biphenyl-, fluorosubstituted terphenyl-, tolane-, and phenyl tolane isothiocyanates are very useful liquid crystalline compounds for diverse applications because of their high polarity, low viscosity, and high birefringence. ${ }^{13-15}$

Electric characterization of the new notch filter is shown and discussed. Filter frequency response has been characterized by measuring the transmission coefficient $S_{21}$ of the scattering matrix vs. microwave frequency. This parameter quantifies the insertion losses of the device and is the decibel (dB) expression of the ratio between the microwave output and input powers.

The experimental setup for measuring the frequency response is depicted in Fig. 3. $\mathrm{S}_{21}$ parameter of the device has been measured by using an 8703B network analyzer from Agilent. Port 1 of the analyzer is coupled to a Bias-T, which is also connected to the input of the notch filter. Next, the filter output is connected to a dc-block input. Finally, the connection of the blocking coupler to the port 2 closes the microwave 


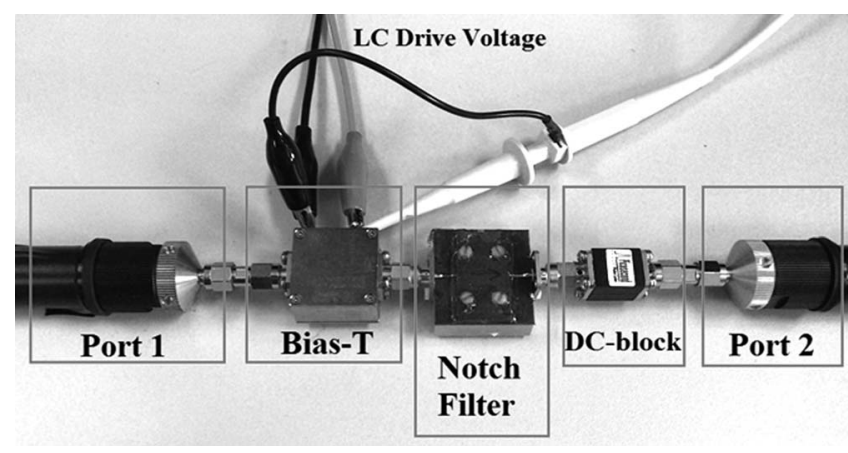

FIG. 3. Experimental setup for measuring the transmission coefficient $S_{21}$ of the scattering matrix vs. microwave frequency.

signal flow towards the network analyzer. The Bias-T consists of a three port network that makes it possible to drive the LC at lower frequencies without disturbing the flow of the microwave frequency signals. Sinusoidal signals of $10 \mathrm{kHz}$ have been applied in order to switch the LC molecules. The intermediate tunable voltage levels were comprised in the range of values from $0 \mathrm{~V}_{\text {rms }}$ to $15 \mathrm{~V}_{\text {rms }}$. A spurline of $1=10 \mathrm{~mm}$ length was chosen for obtaining a filter of about $\mathrm{f}_{0}=5 \mathrm{GHz}$ rejection frequency, from (1) and the effective permittivity considered.

Experimental results of frequency response were obtained for the hypothesis previously described. Frequency de-
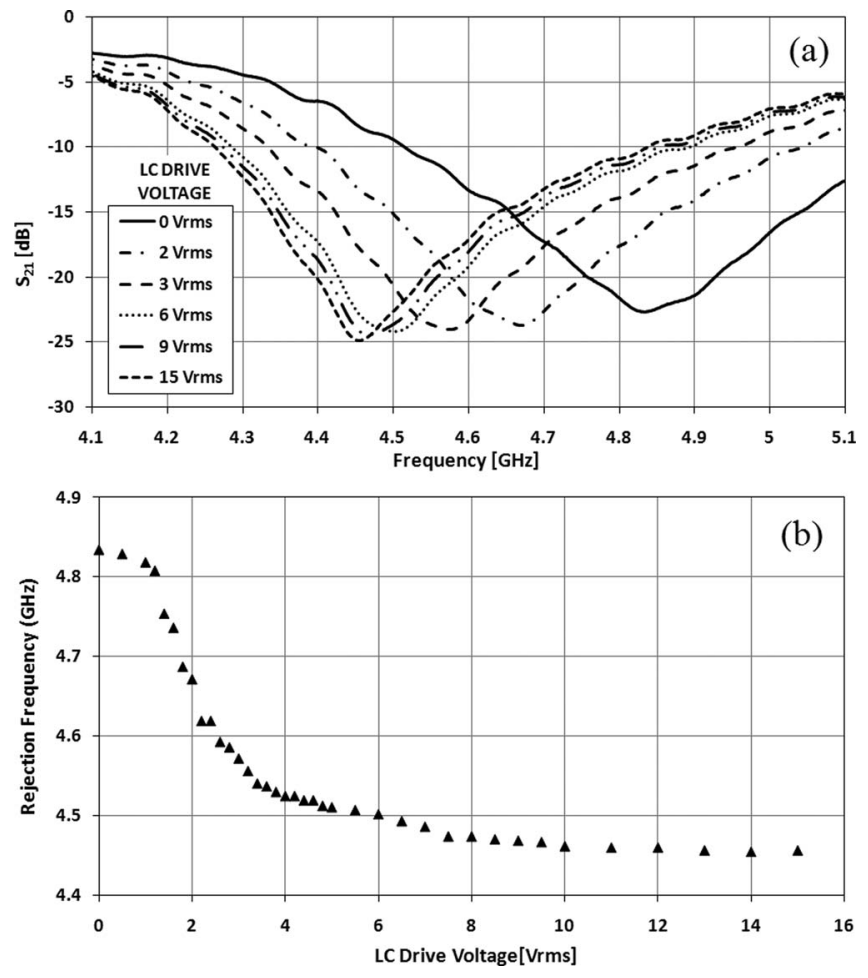

FIG. 4. Experimental results of frequency response and tunability for the notch filter. (a) Frequency dependence of IL for a range of LC driving voltages. (b) LC driving voltage dependence of the filter rejection frequency. pendence of insertion losses for the design filter is graphed in Fig. 4(a). It is shown that, without external voltage, the rejection frequency of the notch filter is about $4.85 \mathrm{GHz}$; molecules exhibit the lower value of permittivity, $\varepsilon_{\mathrm{r} \perp}$. As LC driving voltage increases, permittivity also increases, so rejection frequency decreases. The minimum of the rejection frequency is $4.45 \mathrm{GHz}$, as $15 \mathrm{~V}_{\mathrm{rms}}$ saturation voltage is applied to the filter; permittivity reaches its maximum value, $\varepsilon_{\mathrm{r} \|}$. Therefore, filter tunability relative to central rejection frequency achieves $9 \%\left(\Delta \mathrm{f}_{0} / \mathrm{f}_{0}=0.4 \mathrm{GHz} / 4.65 \mathrm{GHz}\right)$, a very attractive feature. Also, the stopband filter attains a rejection of about $24 \mathrm{~dB}$, a suited value that remains almost constant as voltage increases from 0 to $15 \mathrm{~V}_{\mathrm{rms}}$. Also, range of voltage for the rejection frequency $10 \%-90 \%$, shown in Fig. 4(b), is $4.7 \mathrm{~V}_{\text {rms }}$, just a few volts, therefore it can be generated with standard electronics.

Financial support of the Spanish Ministry of Science and Innovation, Grant No. TEC2009-13991-C02-01, and Comunidad de Madrid, Project No. FACTOTEM2 S2009/ESP1781 , is gratefully acknowledged. The authors wish to thank GFA Group from Universidad Politécnica of Madrid for providing their infrastructures in order to manufacture the LC devices.

${ }^{1}$ K. C. Lim and J. D. Margerum, Proc. SPIE 1815, 99-105 (1992).

${ }^{2}$ B. A. Belyaev, A. A. Leksikov, A. M. Serzhantov, and V. F. Shabanov, Tech. Phys. Lett. 34(6), 463 (2008).

${ }^{3}$ A. Gaebler, F. Goelden, A. Manabe, M. Goebel, S. Mueller, and R. Jakoby, in Proceedings of the 39th European Microwave Conference, Rome, Italy, 29 September-1 October 2009, edited by European Microwave Association (Horizon House Publications Ltd, London, 2009), pp. 594-597.

${ }^{4}$ C. A. Chang, C.-C. Cheng, and J. A. Yeh, IEEE Trans. Electron Devices 53(7), 1675 (2006).

${ }^{5}$ Z.-C. Hao and J.-S. Hong, IEEE Trans. Microwave Theory Tech. 56(9), 2095 (2008).

${ }^{6}$ F. Goelden, A. Gaebler, O. Karabey, M. Goebel, A. Manabe, and R. Jakoby, in Proceedings of the 5th German Microwave Conference, Berlin, Germany, 15-17 March 2010, edited by W. Menzel (University of Ulm, Germany, 2010), pp. 98-101.

${ }^{7}$ S. Bildik, S. Dieter, C. Fritzsch, M. Frei, C. Fischer, W. Menzel, and R. Jakoby, in Proceedings of the 41st European Microwave Conference, Manchester, UK, 10-13 October 2011, edited by European Microwave Association (Horizon House Publications Ltd, London, 2011), pp. 12921295.

${ }^{8}$ K. Chung, S. Hong, and J. Choi, IET Proc. Microwaves, Antennas Propag. 1(2), 518 (2007).

${ }^{9}$ A. Gaebler, A. Moessinger, F. Goelden, A. Manabe, M. Goebel, R. Follmann, D. Koether, C. Modes, A. Kipka, M. Deckelmann, T. Rabe, B. Schulz, P. Kuchenbecker, A. Lapanik, S. Mueller, W. Haase, and R. Jakoby, International Journal of Antennas and Propagation: Active Antennas for Space Applications, 2009, Article ID 876989 (2009).

${ }^{10}$ S. Bulja, D. Mirshekar-Syahkal, R. James, S. E. Day, and F. Aníbal, IEEE Trans. Microwave Theory Tech. 58(12), 3493 (2010).

${ }^{11}$ T. Kuki, H. Fujikake, T. Nomoto, and Y. Utsumi, Electron. Commun. Jpn., Part 2: Electron. 85(2), 36 (2002).

${ }^{12}$ R. N. Bates, IEE J. Microwaves, Opt. Acoust. 1(6), 209 (1977).

${ }^{13}$ R. Dąbrowski, J. Dziaduszek, A. Ziółek, Ł. Szczuciński, Z. Stolarz, G. Sasnouski, V. Bezborodov, W. Lapanik, S. Gauza, and S.-T. Wu, OptoElectron. Rev. 15(1), 47 (2007).

${ }^{14}$ A. Spadło, R. Dąbrowski, M. Filipowicz, Z. Stolarz, J. Przedmojski, S. Gauza, Y. Claire, H. Fan, and S.-T. Wu, Liq. Cryst. 30(2), 191 (2003).

${ }^{15}$ R. Dąbrowski, J. Dziaduszek, K. Garbat, M. Filipowicz, S. Urban, S. Gauza, and G. Sasnouski, Liq. Cryst. 37(12), 1529 (2010). 